\title{
PRÁCTICAS SOCIALES FRONTERIZAS ENTRE CHILE Y BOLIVIA, MOVILIDAD, CIRCULACIÓN Y MIGRACIÓN. SIGLOS XX Y XXI*
}

\author{
Marcela Tapia Ladino** \\ Universidad Arturo Prat (Chile)
}

La región fronteriza del norte de Chile es un territorio histórico de circulación de población boliviana motivada por las posibilidades laborales de la economía regional y las alternativas comerciales existentes en distintos momentos de su devenir. Si bien Tarapacá no ha sido el destino preferente de la migración boliviana, forma parte del campo migratorio especialmente para los aymara que habitan las zonas más cercanas a la frontera y desde los 90 del siglo pasado para los bolivianos en general. Así de una migración fuerte durante el ciclo de expansión del salitre (18801930) se pasó a una más silenciosa y menos visible circunscrita al altiplano y los valles alto andinos para pasar a otra más integrada a lo internacional a partir de la creación de la Zona Franca de Iquique a fines del siglo XX.

Palabras claves: Migración - circulación - región fronteriza - movilidad

SOCIAL BORDER PRACTICES BETWEEN CHILE AND BOLIVIA: MOBILITY, CIRCULATION AND MIGRATION IN THE $20^{\text {TH }}$ AND $21^{\text {ST }}$ CENTURIES

Chile's northern border region has historically been a territory of circulation for the Bolivian population that has been motivated by job opportunities and trade alternatives in the regional economy. While Tarapacá has not been the main destination of Bolivian migration, it has formed part of the migratory field, especially for the Aymara, who live in the areas closest to the border, and for Bolivians in general since the 1990s. From strong migration during the saltpeter expansion cycle (1880-1930), the area moved to a more silent and less visible migration limited to the altiplano and upper Andean valleys and then to a process that was more integrated into the international context beginning with the creation of the Tax Free Zone in Iquique at the end of the 2oth century.

Keywords: Migration, circulation, border region, mobility

Articulo Recibido: 15 de Abril de 2018

Artículo Aceptado: 12 de Mayo de 2018

\footnotetext{
* Este trabajo es resultado de FONDECYT Regular 1150123 (2015-2018) y FONDECYT Regular 1171722 (2017-2020).

**E-mail: : marcela.tapia@unap.cl
} 
$\mathrm{E}$ n las últimas décadas Chile asiste a un crecimiento de la inmigración de origen sudamericano y caribeño lo que ha llamado la atención de los cientistas sociales y de la clase política produciendo numerosos estudios y movimientos pro y anti migrantes ${ }^{1}$. Sin embargo, el aumento de las migraciones hacia Chile es parte de un fenómeno mundial que indica que no se detendrán en el corto plazo y que por contrario irán en aumento ${ }^{2}$. Dentro de la variedad de formas que adquiere la migración, la fronteriza es una de las más frecuentes debido a la cercanía entre países y regiones colindantes. La motivación para migrar a países fronterizos es fundamentalmente económica, animada por las facilidades de cruce que da la contigüidad fronteriza, el efecto llamada del mercado laboral regional y las diferencias de desarrollo a uno y otro lado de la frontera que permiten aprovechar el tipo de cambio y retornar periódicamente. En América Latina existen prácticas de circulación de población que tienen su correlato en historia de las regiones fronterizas y que se han constituido en foco de interés de distintas disciplinas en el último tiempo ${ }^{3}$. Recientemente Chile y Brasil se suman a Argentina

\footnotetext{
${ }^{1}$ Stefoni, C. y Stang, F., «La construcción del campo de estudio de las migraciones en Chile: notas de un ejercicio reflexivo y autocrítico", Íconos-Revista de Ciencias Sociales, n58, 2017 (pp. 109-129).

2 El Informe Mundial de Migraciones 2018 señala que las proyecciones de crecimiento de las migraciones realizadas el año 2003 para el 2050 fueron sobrepasadas ya el año 2015. Asimismo, señala que el comportamiento de las migraciones si bien es volátil, tiende a aumentar en las migraciones internas y transfronterizas, especialmente estas últimas producto de conflictos civiles y transnacionales, Mcauliffe, M. y Ruhs, M., World Migration Report 2018, OIM, Switzerland, 2017, p. 3.

3 Benedetti, Alejandro y Salizzi, E., «Llegar, pasar, regresar a la frontera. Aproximación al sistema de movilidad argentino-boliviano», Transporte y territorio, n4, 2011 (pp. 148-179); Domenach, H., et al., Movilidad y procesos migratorios en el espacio fronterizo Argentino-Bolviana, Editorial Copiar, Córdoba, 2007; Tapia, Marcela, «Migración y movilidad de los trabajadores fronterizos en Tarapacá durante el ciclo del nitrato. 1880-1930», Ed. González, S., La sociedad del salitre: protagonistas, migraciones, cultura urbana y espacios públicos, 1870-1940, RIL, Santiago de Chile, 2013 (pp. 163-194).
} 
como destino migratorio, especialmente de extranjeros de origen fronterizo en el marco de la crisis del sur de Europa y de Estados Unidos ${ }^{4}$.

En la historia de Chile la migración fronteriza tiene larga data ${ }^{5}$, especialmente a fines del siglo XIX y entrado el siglo XX cuando se incorporan los territorios de Tarapacá y Antofagasta al territorio nacional como resultado de la Guerra del Pacífico (1879 1881) ${ }^{6}$. Así nuestro país comparte una extensa frontera, especialmente con Argentina y Bolivia y, en el caso de Perú la frontera es menos extensa, pero el paso fronterizo de Chacalluta es la principal puerta de entrada terrestre de la migración reciente de origen sudamericano y caribeño ${ }^{7}$. Por tanto, no es extraño que la migración peruana y boliviana tenga mayor profundidad histórica, especialmente en el norte de Chile dada la cercanía y la atracción que ejerce la economía regional en el entorno (Tapia, 2012). Sin embargo, el estudio la movilidad humana en un región fronteriza requiere de una mirada histórica que supere las interpretaciones coyunturales -la limitación de la época ${ }^{8} \sim$ que en ocasiones tienden a predominar en los análisis y a sobredimensionar los hallazgos de investigación. Por tanto el propósito de este artículo es ampliar la perspectiva histórica del fenómeno migratorio de acuerdo a los aportes de la Historia del Tiempo Presente que entiende la preocupación por los hechos recientes como atención o como “... la ‘visión’ que hace de nuestro espacio de experiencia, nuestro campo de observación y de investigación"9. En este sentido se reconoce que la noción del presente contiene una mirada hacia delante y hacia atrás, en tanto se acepta que "el único conocimiento que podemos esperar alcanzar es de carácter histórico: nuestro presente es historia" ${ }^{10}$. Se ha discutido y revisado la noción tradicional de la historia apegada al relato de los hechos -fundamentalmente políticos y de los grande líderes y se ha avanzado durante el siglo XX hacia la incorporación de nuevas formas de hacer historia ${ }^{11}$, a través de la inclusión de herramientas metodológicas diversas y la preocupación por el quehacer humano en un sentido amplio ${ }^{12}$. Así el afán de este artículo es aportar, desde la historia y con las contribuciones de disciplinas afines, como la geografía y las

\footnotetext{
${ }^{4}$ Texidó, Exequiel y Gurrieri, J., Panorama migratorio de América del Sur 2012, Organización Internacional para las migraciones OIM, Buenos Aires, 2012.

${ }^{5}$ Norambuena, C., Migraciones limítrofes. Chile 1865-1960, en Asdrúbal, H. (ed.), Historia de las migraciones limítrofes en el Cono Sur de América. Argentina, Bolivia, Brasil, Chile, Paraguay y Uruguay. Vol II Bolivia, Chile, Paraguay y Uruguay, Instituto Panamericano de Geografía e Historia, México D.F., 2002 (pp. 25-90).

${ }^{6}$ González, S., «El Norte Grande de Chile: La definición histórica de sus límites, zonas y líneas de fronteras y la importancia de las ciudades como geosímbolos fronterizos», Revista de Historia Social y de las Mentalidades, $\mathrm{n}^{\circ} 13$ (2), 2009 (pp. 9-42); González, S., La llave y el candado: el conflicto entre Perú y Chile por Tacna y Arica (1883-1929), LOM, Santiago de Chile, 2008.

7 DEM, Migración en Chile 2005-2014, Sección Estudios del Departamento de Extranjería y Migración, Ministerio del Interior, Santiago de Chile, 2016, p. 46.

${ }^{8}$ Burke, Peter, Sociología e Historia, Alianza Editorial, Madrid, 1987, p. 12.

9 Bédarida, Francois, «Definción, método y práctica de la Historia del Tiempo Presente», Cuadernos de Historia Contemporánea, n²0, 1998 (pp. 19-27), p. 22

${ }^{10}$ Bertaux, Daniel, «Desde la perspectiva de la historia de vida a la transformación de la práctica sociológica», en Marinas, J. M. y. C. S. (eds.), La Historia oral: métodos y experiencias, Debate, Madrid, 1993 (pp. 19-34), p. 25.

${ }^{11}$ Burke, Peter, Formas de hacer historia, Alianza Editorial, Madrid, 2009.

${ }^{12}$ Ariostegui, Julio, «Historia y Tiempo Presente. Un nuevo horizonte de la historiografía contemporaneista», Cuadernos de Historia Contemporánea, n²0, 1998 (pp.15-18); Sauvage, Pierre, «Una historia del tiempo presente», Historia Crítica, n 17, 1998 (pp. 59-70); Rioux, Jean Pierre, «Historia del Tiempo Presente y demanda social», Cuadernos de Historia Contemporánea, n²0, 1998 (pp. 71-80).
} 
teorías migratorias, a un conocimiento más amplio de la movilidad a través de la inclusión de una mirada diacrónica y regional de los movimientos de población.

El objetivo de este trabajo es conocer las especificidades que adquiere el desplazamiento de bolivianos a la región de Tarapacá situada al norte de Chile en un contexto la larga duración, es decir, desde principios del siglo XX a principios del siglo XXI. Asimismo, interesa revisar lo que hemos denominado prácticas sociales fronterizas, es decir, las actividades que tienen como referente a la frontera, especialmente respecto de la movilidad, formas de cruce, los motivos e incentivos para moverse de un territorio a otro, así como la circulación. El escenario es el norte de Chile (Tarapacá y Arica-Parinacota) y occidente boliviano y el foco son las prácticas de movilidad que no siempre tienen como fin el establecimiento en el lugar destino, sino cruzar la frontera para aprovechar las ventajas del cruce con fines laborales y comerciales. El periodo de estudio es el siglo XX con especial énfasis los años 90 del siglo pasado hasta entrado el siglo XXI.

Mapa $N^{\circ} 1$ Región fronteriza. Norte de Chile, sur peruano y occidente boliviano

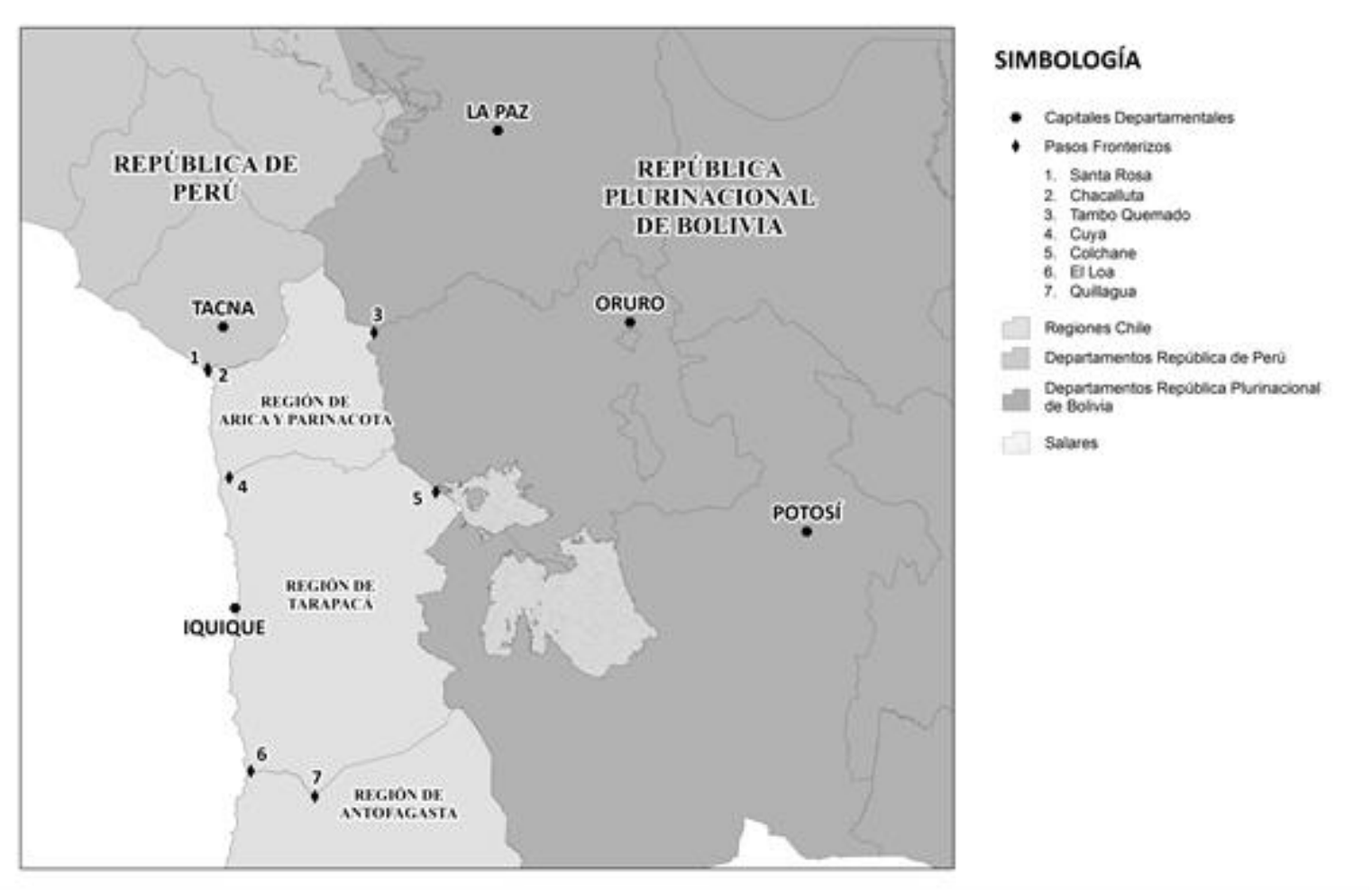


Un aspecto a tener en cuenta es la dificultad para relevar algunas categorías que se encuentran a menudo subsumidas en las etiquetas nacionales, por ejemplo, la adscripción étnica. En general los censos del siglo XX incluyeron tardíamente el origen étnico de los encuestados y, por tanto, se trata de información difícil de estimar, especialmente para principios del siglo XX. Como señalamos en otra parte, durante el periodo del salitre "debajo de esa pátina obrera y nacional quedó invisibilizada la presencia indígena que, sin embargo, se manifestó en algunas festividades pampinas como el Carnaval, la Cruz de mayo y la Fiesta de la Virgen del Carmen de La Tirana (Díaz, 2009)"13. En un sentido similar, cuando los antropólogos se dedicaron a estudiar las comunidades andinas, especialmente en los años 60 y 70, pocas veces dieron cuenta de la nacionalidad y en general se estudió a los aymara sin reparar mayormente en el origen nacional. Bajo el influjo del desarrollismo se tendió a conceptualizarlos como campesinos de origen andino con menor detenimiento en las trayectorias migratorias o lugares de origen. Por tanto, teniendo en cuenta estas limitaciones de las fuentes estadísticas y secundarias es que abordamos el estudio de la presencia boliviana en Tarapacá con el afán de aportar al conocimiento de las migraciones a nivel regional y nacional.

Para el análisis hemos considerado los aportes de los estudios de frontera (border studies), la geografía social francesa y el paradigma de la movilidad de Alain Tarrius ${ }^{14}$. Como fuente hemos utilizado la prensa local, La Estrella de Arica y La Estrella de Iquique en una revisión que abarcó 17 años, desde 1990 hasta el 2010. Asimismo, utilizamos fuentes secundarias entre ellos informes de organismo gubernamentales, tesis de pre y posgrado, bibliografía específica, así como datos estadísticos sobre extranjeros elaborados por el Instituto nacional de Estadística y del Ministerio del Interior.

El artículo se organiza en tres partes, la corresponde al marco conceptual que guía el análisis de la movilidad humana en Tarapacá histórico (anterior a la división del 2007) a partir de los aportes de los estudios fronterizos a la historia. La segunda parte corresponde a una revisión de la presencia boliviana en la región durante el siglo XX. Para eso distinguimos el periodo del salitre, mediados del siglo XX y entrado los años 90 y las variaciones a nivel regional de acuerdo con la historia de Arica e Iquique fundamentalmente. La tercera parte corresponde a las estrategias, motivos y formas de cruce de la frontera que se identifican en la prensa desde los años 90 en adelante en el marco del aumento de las migraciones fronterizas hacia Chile.

\section{Las regiones fronterizas para el estudio de la movilidad humana}

Los estudios fronterizos (border studies) han experimentado cambios durante el siglo XX al pasar de la preocupación por los límites en clave geopolítica hasta la primera mitad de la centuria, a las posturas más optimistas que plantearon el fin de las fronteras en las últimas décadas

\footnotetext{
13 Díaz, A. y Tapia, M., «Los aymara del norte de Chile entre los siglos XIX y XX. Un recuento histórico», Atenea, $\mathrm{n}^{\circ}$ 507, 2013 (pp. 181-196), p. 189.

14 Tarrius, Alain, «Au-delà des États-nations : des sociétés de migrants», Revue européenne de migrations internationales, $n^{\circ}$ 17(2), 2001 (pp. 37-61); Tarrius, Alain, La mundialización por abajo. El capitalismo nómada en el arco mediterráneo, Hacer Editorial, Barcelona, 2007, Tarrius, Alain, «Pobres en migración, globalización de las economías y debilitamiento de los modelos integradores: el transnacionalismo migratorio en Europa meridional», Empiria. Revista de metodología de Ciencias Sociales, n 19, 2010 (pp. 133-156).
} 
del siglo15 y a un desencanto de las dada el alcance limitado del "mundo sin fronteras". El debate sigue en curso y en la actualidad una de las líneas de investigación propone comprender a las fronteras como la suma de procesos sociales, culturales y políticos más que como demarcadores o líneas fijas ${ }^{16}$. Así el debate sobre las fronteras se ha ampliado en tanto se acepta que éstas ya no se circunscriben sólo a la línea trazada en el mapa sino que se encuentran en distintas partes, aeropuertos, embajadas y controles biométricos, en el marco de la lucha contra el terrorismo, los movimientos anti inmigración y los procesos de securitización asociados .

En general la discusión sobre las fronteras está contenida en casi todas las disciplinas sociales desde la geografía a la teoría política, pasando por la antropología, la historia y la sociología, por tanto, el objetivo aquí es sintetizar los derroteros de este debate para el estudio de la movilidad en una región fronteriza privilegiando la importancia en la historia. De manera general la discusión se mueve entre el énfasis en los procesos de interacción e integración hasta aquellos que se centran en el cierre y la fronterización, en los cuales los movimientos de población ocupan un lugar central y son los que padecen las consecuencias. Asimismo, los enfoques varían desde aquellos que se sitúan desde arriba hacia abajo o en el sentido inverso, en cualquier caso existe bastante consenso sobre la necesidad de incluir el análisis multiescalar o multinivel en los estudios sobre las fronteras ${ }^{17}$ sin perder de vista las "viejas jerarquías", como lo nacional, que no han desaparecido del todo y siguen teniendo injerencia, especialmente a nivel territorial ${ }^{18}$.

En esta discusión las regiones fronterizas se constituyen en espacios privilegiados para observación de la movilidad humana y el lugar que ocupa la frontera en las prácticas de cruce, circulación y retorno. Para este trabajo adscribimos al concepto de región fronteriza definido como un espacio de "desarrollo de un conjunto de actividades al interior de cada estado y que tienen a la frontera como un centro vital de referencia"19. Así las regiones fronterizas son zonas donde se sintetizan las acciones de convergencia de espacios contiguos -dos o más Estados , pero que son también espacios de contradicción, es decir, que oscilan entre la interacción y la restricción, apertura y cierre. Por tanto, esta noción contiene una definición de frontera que no incluye sólo el límite -la línea sino a toda la zona delimitada por ésta donde "en la yuxtaposición de lo espacial y lo lineal estriba el origen de la diferenciación. La frontera es territorio compartido y el límite una línea imaginaria que lo divide jurídicamente" ${ }^{20}$. Así luego de un debate que sigue en curso, la comunidad científica ha dejado de ver las fronteras como entes estáticos o simples líneas, para pasar a comprenderlas como procesos y realidades construidas en permanente movimiento donde la región

\footnotetext{
${ }^{15}$ Tapia, Marcela y González, A., Presentación. «Fronteras, regiones fronterizas y migraciones. Entre apertura, integración y cierre», en Tapia, Marcela. y González, A. (Eds.), Regiones fronterizas, migración y los desafíos para los Estados nacionales latinoamericanos, RIL Editores, Santiago de Chile, 2014 (pp. 15-38).

${ }^{16}$ Johnson, Corey, et al., «Interventions on rethinking 'the border' in border studies», Political Geography, $\mathrm{n}^{\circ} 30$ (2), 2011 (pp. 61-69).

17 Jessop, Bob, «La economía política de la escala y la construcción de las regiones fronterizas», EURE, $n^{\circ} 30$ (89), 2004 (pp. 25-41).

${ }^{18}$ Sassen, Saskia, Territorio, autoridad y derechos. De los ensamblajes medievales a los ensamblajes globales, Katz Editores, Madrid, 2010.

${ }^{19}$ Morales, Abelardo, «Desentrañando fronteras y sus movimientos transnacionales entre pequeños estados. Una aproximación desde la frontera Nicaragua-Costa Rica», en Anguiano, M. E. y López, A. (eds.), Migraciones y frontera. Nuevos contornos para la movilidad internacional, Icaria, Barcelona, 2010 (pp. 185-224), p. 187.

${ }^{20}$ Nweihed, Kaldone, Frontera y límite en su marco mundial: una aproximación a la "fronterología", Ediciones de la Universidad Simón Bolívar, Caracas, 1992, pp. 28-29.
} 
fronteriza se constituye en la unidad de análisis ${ }^{21}$. Sin embargo, es preciso tener en cuenta que, a pesar del interés por la interacción, estas regiones siguen siendo el recordatorio de las consecuencias sociales y políticas del proceso de definición y redefinición de los límites, especialmente notorio en América Latina y en la triple frontera que comparte Chile, Bolivia y Perú.

En América Latina las fronteras estatales son de reciente configuración en términos históricos, aunque la delimitación se inició con los procesos de emancipación en las primeras décadas del siglo XIX éstas no se terminaron de establecer sino hasta entrado el siglo XX. Este proceso implicó conflictos y litigios, muchos de los cuales tienen impacto hasta el día de hoy. Por tanto, la comprensión de los fenómenos como la movilidad, que tiene como referente a la frontera implica no olvidar el carácter recordatorio de los lindes. Si bien no nos detendremos en el debate diplomático que ha dado lugar la delimitación señalada la tendremos en cuenta en tanto sea un factor de comprensión en el análisis.

Para comprender las formas que adquiere los movimientos de población desde Bolivia hacia Tarapacá es pertinente la utilización del concepto de movilidad que es más amplio que el concepto de migración y de hecho establece que la migración es una forma más de movilidad ${ }^{22}$. Básicamente la movilidad se define respecto del motivo, el tiempo y el lugar, es decir, se trata de motivos específicos (laboral, comercial, salud, compras, etc), son de corta duración, aunque pueden se reiterados en el tiempo y lo más importante es que no implican un cambio de residencia ${ }^{23}$. La ventaja de este concepto, respecto al de migración, es que hace menos juicios acerca de la naturaleza de los desplazamientos humanos y de sus fines. Este último alude normalmente al establecimiento con el objetivo central del desplazamiento y el retorno como el fin del ciclo una vez conseguido el éxito económico en el país de destino. No se trata de fenómenos nuevos, sino de la necesidad de relativizar conceptos que no dan cuenta suficientemente de las distintas formas de moverse en el espacio y de la inadecuación de las herramientas conceptuales y metodológicas para captar esa "ambigüedad migratoria"24 o las categorías residuales de la migración ${ }^{25}$. Asimismo, la evidencia empírica invita a dejar de concebir las migraciones como rupturas espacio temporales, para mirar las conexiones y vínculos que establecen quienes migran o se mueven, y así atender a la circulación de personas como una forma de movilidad. De acuerdo con estos aportes es posible mirar las dinámicas que producen y definen las circulaciones, así como las modalidades que adquieren, los actores "conexos" (no migrantes, pasadores, transportistas) y los "recursos circulatorios" es decir, al saber y poder circular de quienes

\footnotetext{
${ }^{21}$ Zapata-Barrero, Ricard y Ferrer-Gallardo, X., «Las fronteras en la época de la movilidad», en Zapata-Barrero, Ricard y Ferrer-Gallardo, X. (eds.), Fronteras en movimiento. Migraciones hacia la Unión Europea en el contexto Mediterráneo, Edicions Bellaterra, Barcelona, 2012 (pp. 11-56), p. 13.

${ }^{22}$ Heyman, J., «Construcción y uso de tipologías: movilidad geográfica en la frontera México-Estados Unidos», en Ariza, M. y Velasco, L. (eds.), Métodos cualitativos y su aplicación empírica. Por los caminos de la investigación sobre migración internacional, Instituto de Investigaciones Sociales-UNAM/El Colegio de la Frontera Norte, México D.F., 2012 (pp. 419-454).

${ }^{23}$ Castillo, M. y Nájera, J., «México como país de origen, tránsito y destino de migrantes, una revisión a partir de la emif norte y la emif sur», en Consejo Nacional de Población (ed.), 20 años de la Encuesta sobre Migración en la Frontera Norte de México, 2014 (pp. 17-35).

${ }^{24}$ Cortés, Genevieve, «Migraciones, construcciones transnacionales y prácticas de circulación. Un enfoque desde el territorio», Párrafos geográficos, n 8 (1), 2009 (pp. 35-53), p. 38.

${ }_{25}$ Mallimaci, Ana Inés, «Movilidades y permanencias. Repensando la figura del movimiento en las migraciones», Revista de Temas de Antropología y Migración, 2012 (pp. 77-92).
} 
se mueven ${ }^{26}$ y al sentido que dan a esas prácticas espaciales.

Desde el punto de vista histórico las movilidades se han desarrollado con más claridad una vez conformados los Estados nacionales, pero regularmente se les definió como migración y hubo poca atención a los movimientos o idas y venidas de las personas que no se terminaban de asentar en un sitio. La historiografía tipificó estos movimientos como migración temporal o pendular casi siempre de tipo laboral. En Chile, estos movimientos se han estudiado en el norte durante el ciclo de expansión del salitre ${ }^{27}$ y en el sur especialmente de trabajadores chilotes que viajaban a la Patagonia a trabajar ${ }^{28}$. Muchos de estos movimientos dieron por resultado la migración, es decir, el asentamiento en el lugar de destino, pero en otros casos cuando se acabó la bonanza y los trabajadores regresaron a su lugar de origen.

\section{Tarapacá en el campo migratorio boliviano, entre migración y circulación}

Al revisar la historia migratoria de Bolivia apreciamos que Chile no ha sido el destino migratorio preferente de dicho país ${ }^{29}$, sino que ésta se ha dirigido fundamentalmente a Argentina durante casi todo el siglo XX y a fines de la centuria a Estados Unidos y entrado el siglo XXI a Europa y en mucho menor medida a nuestro país ${ }^{30}$. Sin embargo, podemos afirmar que el norte de Chile sí tiene un lugar en el campo migratorio boliviano, si bien marginal es constante en el tiempo y relacionado con los ciclos de la economía regional. En otro estudio constatamos que la presencia de los extranjeros de origen fronterizo, es decir, peruano y boliviano, ha sido constante durante toda la historia regional del siglo XX y por sobre la media nacional ${ }^{31}$. Por tanto, es posible afirmar que la migración boliviana a Chile ha sido una migración silenciosa, menos visible en tanto posee mayor profundidad histórica y ya es parte innegable de la vida tarapaqueña.

Al término de la Guerra del Pacífico las comunidades andinas fronterizas de Tarapacá fueron, en varios casos, divididas por los nuevos límites trazados de acuerdo con el Tratado de 1904 además de la distinción de los habitantes entre chilenos o bolivianos ${ }^{32}$. De hecho "las comunidades aymaras, así como toda la población tarapaqueña, debieron elaborar una nueva relación con el Estado, ahora como "nuevos chilenos" a través de la inscripción de las propiedades, el reclutamiento militar y la educación, proceso que no estuvo exenta de

\footnotetext{
${ }^{26}$ Cortés, Genevieve, op. cit.

${ }_{27}$ González, S., «Arrieros argentinos en el ciclo salitrero de Tarapacá», Revista de Estudios Trasandinos, n 4, 2000 (pp. 117-129); González, S., "Cruzando los mallkus. Las migraciones bolivianas pendulares durante las grandes crisis salitretas (1914-1933)", Revista de Historia Social y de las Mentalidades, n², 2006 (pp. 155-191).

${ }^{28}$ Saldivar, J. M., «"Chilote tenía que ser": Vida migrante transnacional en territorios patagónicos de Chile y Argentina», CUHSO Cultura-Hombre-Sociedad, n² 27 (2), 2017 (pp. 175-200); Arias, F., «Los pueblos del noroeste de la Patagonia Argentina. Relaciones históricas y etnológicas con el sur continental chileno y el Archipielago de Chiloé. Siglo XVII-XVIII», Revista de Historia, n 9, 2014 (pp. 45-70).

${ }^{29}$ Tapia, Marcela, «Bolivia, historia de migraciones: pasado y presente», en Solé, C., et al. (eds.), Las migraciones bolivianas en la encrucijada interdisciplinar: Evolución, cambios y tendencias. CER Migraciones/Universitat Autònoma de Barcelona, Barcelona, 2014 (pp. 9-29).

${ }^{30}$ Texidó, E. y Gurrieri, J., op. cit.

${ }^{31}$ Tapia, Marcela, «Frontera y migración en el Norte de Chile a partir del análisis de los censos de población. S. XIX y XXI», Revista Geografía Norte Grande, n 52, 2012 (pp. 177-198).

${ }_{32}$ González, S., «La presencia indígena en el enclave salitrero de Tarapacá: Una reflexión en torno a la fiesta de La Tirana», Chungará, Revista de Antropología Chilena, n 38 (1), 2006 (pp. 35-49).
} 
disputas y litigios con el Estado chileno ${ }^{33}$. Sin embargo, las comunidades que habitaban la frontera a menudo ignoraron el linde en tanto límite geopolítico y lo cruzaron constantemente para distintas actividades como pastoreo, comercio o visitas a familiares ${ }^{34}$. Sin embargo, la presencia urbana de bolivianos se mantuvo, prueba de ello es la Sociedad Boliviana de Socorros Mutuos de Iquique fundada el 14 de septiembre de 1893, que con altos y bajos sigue vigente hasta el día de hoy. Así desde fines del ciclo del salitre la presencia boliviana la podemos distinguir entre migrantes en el sentido clásico, es decir, de personas que se quedaron en la región después de la crisis he hicieron su vida en Arica, Iquique o en los pueblos de los oasis y precordilleranos. También durante este periodo las fuentes mencionan a personas que siguieron cruzando la frontera para trabajar, pastorear o comerciar y que no necesariamente se asentaron y si lo hicieron no dejaron de ir y venir como revisaremos más adelante.

\section{Presencia de bolivianos en Tarapacá en la primera mitad del siglo XX}

La presencia boliviana en la región tiene larga data y el vínculo remite a la época del salitre, periodo en el que la importancia de la presencia altiplánica, tanto en términos absolutos como relativos fue notoria durante todo el periodo y se expresó en la incorporación de hábitos, expresiones culturales y productos de consumo ${ }^{35}$. El boom salitrero motivó la llegada de los trabajadores bolivianos la que se realizó por largos y penosos recorridos gracias al enganche y acción de arrieros que facilitaban el cruce cordillerano. "El cruce requería de toda una logística que incluyó a arrieros conocedores de la cordillera, vaqueanos que sirvieron de guía y de recuas de mulas para el traslado de pertenencias"36.

Así la presencia de bolivianos en el norte salitrero de Chile se caracterizó por la estacionalidad asociada al ciclo del nitrato y luego una disminución debido a la crisis del salitre de los años 30, rasgo que compartieron todos los grupos extranjeros que se instalaron en la zona durante el ciclo de expansión del salitre. Una vez acabada la bonanza decae la llegada de bolivianos en su mayoría de origen aymara, pero no desaparece y se mantiene a través del despliegue de una serie de prácticas de cruces y circulaciones, que revisaremos más adelante, hasta la segunda mitad del siglo XX sin mayores dificultades más allá de los geográficos y la falta de conectividad (Ver Tabla $\mathrm{N}^{\circ}$ 1).

\footnotetext{
33 Díaz, A. y Tapia, Marcela, op. cit.

34 González, S., «La hoja transfronteriza. El consumo de coca en las faenas mineras salitreras en el Norte Grande de Chile (1900-1930)», Historia Crítica, n59, 2016; González, S., Hombres y mujeres de la pampa. Tarapacá en el ciclo de expansión del salitre, LOM, Santiago de Chile, 2002.

35 Idem.

${ }^{36}$ Tapia, Marcela, Migración..., op.cit., p. 176.
} 
Tabla № 1. Bolivianos en Tarapacá por censos 1885 2002

\begin{tabular}{|c|c|c|c|c|c|}
\hline Censos & Bolivianos & $\begin{array}{c}\text { Total } \\
\text { Extranjeros. } \\
\text { Provincia de } \\
\text { Tarapacá }\end{array}$ & $\begin{array}{c}\text { \% Bolivianos } \\
\text { respecto del } \\
\text { total de } \\
\text { extranjeros }\end{array}$ & $\begin{array}{c}\text { Total } \\
\text { Población } \\
\text { de } \\
\text { Tarapacá }\end{array}$ & $\begin{array}{c}\text { \% Bolivianos } \\
\text { respecto del } \\
\text { total de pobl. } \\
\text { de la } \\
\text { provincia de } \\
\text { Tarapacá }\end{array}$ \\
\hline $\mathbf{1 8 8 5}$ & 4.581 & 23.384 & 19,6 & 45.086 & 10,2 \\
\hline $\mathbf{1 8 9 5}$ & 5.817 & 25.544 & 22,8 & 89.751 & 6,5 \\
\hline $\mathbf{1 9 0 7}$ & 12.528 & 43.774 & 28,6 & 110.036 & 11,4 \\
\hline $\mathbf{1 9 2 0}$ & 5.887 & 15.422 & 38,2 & 100.553 & 5,9 \\
\hline $\mathbf{1 9 3 0}$ & 4.643 & 13.598 & 34,1 & 113.331 & 4,1 \\
\hline $\mathbf{1 9 4 0}$ & 3.403 & 7.125 & 47,8 & 104.097 & 3,3 \\
\hline $\mathbf{1 9 5 2}$ & 2.355 & 6.047 & 38,9 & 102.789 & 2,3 \\
\hline $\mathbf{1 9 6 0}$ & 4.831 & 6.118 & 79,0 & 123.070 & 3,9 \\
\hline $\mathbf{1 9 8 2} *$ & 2.929 & 5.096 & 57,5 & 275.144 & 1,1 \\
\hline $\mathbf{1 9 9 2} * *$ & 3.059 & 6.084 & 50,3 & 339.579 & 0,9 \\
\hline $\mathbf{2 0 0 2}$ & 5.045 & 12.793 & 39,4 & 428.594 & 1,2 \\
\hline
\end{tabular}

*Corresponde a extranjeros totales. Incluye residentes y transeuntes. Es imposible filtrar sólo a residentes habituales dentro del país en esta base de datos.

**A partir del censo 1992 corresponde sólo a extranjeros residentes.

Por el Tratado de Lima (1929) Arica se incorporó al territorio nacional de manera definitiva, hecho que marcó el devenir de las nuevas provincias cuyo trazado afectó a las comunidades fronterizas ${ }^{37} \mathrm{y}$ creó un límite siguiendo en gran parte al trazado del ferrocarril Arica $\mathrm{La} \mathrm{Paz}^{38}$. Iquique pasó a ser capital regional y ambas provincias (Arica e Iquique) se sumieron en una crisis económica que sólo superó Arica en la década de 60 gracias a la creación del Puerto Libre (1953) y la Junta de Adelanto (1958 1976) ${ }^{39}$ y sólo temporalmente sorteada en Iquique por el desarrollo de la industria pesquera en los años $60^{40}$. Así, la atracción que ejerció Arica gracias a su crecimiento incentivó la migración de la población aymara chilena a la ciudad sin abandonar sus tierras, sino ampliando y complejizando los límites de la comunidad. La producción agrícola,

37 González, S. y Ovando, C., «Sama y Camarones: Las fronteras que no fueron entre Perú y Chile», Revista de geografía Norte Grande, $n^{\circ}$ 66, 2017 (pp. 61-82).

${ }^{3}$ Idem.

39 Galdames, L. y Ruz, R., «La junta de adelanto de Arica y John V. Murra: dos lecturas sobre el desarrollo andino en el norte de Chile», Chungará (Arica), n 42 (1), 2010 (pp. 257-270).

${ }^{40}$ Guerrero, B., «La ciudad y sus transformaciones: memoria urbana de Iquique», Revista de Ciencias Sociales, $\mathrm{n}^{\circ} 2$ (19), 2007 (pp. 149-165). 
el abastecimiento hortícola de Arica, el transporte en camiones y la economía informal fueron las vías por las que se mantuvo el vínculo con los valles ${ }^{41}$. Así la migración rural urbana, aunque no puso fin a la vida en el interior, impulsó a la población aymara boliviano a moverse hacia el área más cercana a la frontera para trabajar, pastorear y comerciar. Se trató de la migración escalonada de aymara bolivianos que progresivamente ingresaron a la región desde las zonas colindantes del límite a la Provincia de Parinacota con fines laborales, especialmente a los valles y costa ${ }^{42}$. Allí se incorporaron al mercado laboral agrícola de los valles medio y alto andinos en la medida que se impulsó el desarrollo agrícola que atrajo de mano de obra para el cultivo de chacras o labores de pastore ${ }^{43}$. Las ciudades también ejercieron un poder atractor, especialmente en el caso de Arica bajo la acción de la Junta de Adelanto.

Es preciso señalar que los movimientos de población aymara boliviano no tienen sólo fines laborales, sino también comerciales lo que ha dado origen a la naciente clase alta o ricos aymaras (qamiris) ${ }^{44}$ y de pequeños comerciantes que progresivamente encontraron en el cruce mayores posibilidades de comercio. La condición marginal de Oruro, la pobreza y la baja presencia del Estado o "falsa presencia del "Estado aparente" ${ }^{45}$ durante los años 50 en Bolivia impulsaron a los comerciantes aymara de ese país a buscar oportunidades en Chile. Fue la época de las caravanas de amistad (1958) entre Iquique y Oruro momento en que ambas ciudades buscaron construir un camino que las uniera. Aunque se trató de un proyecto fallido aún está en la memoria regional la llegada los orureños a Iquique (21 de mayo) y luego el de iquiqueños a Oruro (5 de agosto) en la búsqueda de la ansiada conectividad que sólo se concretó a fines del siglo $\mathrm{XX}^{46}$. A pesar de la falta de buenos caminos y como señala Llanque "Los qamiris orureños ingresaron a la modernidad de las puertas de su contacto con Chile, precisamente porque los principales productos para su subsistencia así como sus actividades comerciales las realizaban con este país, pues el eje y el Estado centralizado no les otorgaban absolutamente nada, sino por el contrario les exigía tributos y otro tipo de deberes"47. La contigüidad fronteriza, la bonanza y la caída de la minería del estaño y la ausencia del Estado en departamento de Oruro propiciaron una relación más cercana con el norte de Chile especialmente a través del comercio y el abastecimiento de productos de primera necesidad, a pesar de las dificultades en el acceso.

En el norte de la región y en el marco del impulso de Arica por la creación del Puerto Libre (1953) y más tarde a la Junta de Adelanto (1958) incentivaron la migración campo ciudad de aymara chilenos. Asimismo, la llegada de diversas mercancías impulsó a los aymara bolivianos a comprar en Arica productos de primera necesidad como harina. Como señala un testimonio

\footnotetext{
${ }^{41}$ Gundermann, H., «Procesos regionales y poblaciones indígenas en el norte de Chile. Un esquema de análisis con base en la continuidad y los cambios de la comunidad andina», Estudios Atacameños, n²1, 2001 (pp. 89-112).

${ }^{42}$ Tapia, Marcela, «Frontera, movilidad y circulación reciente de peruanos y bolivianos en el norte de Chile», Estudios Atacameños. Arqueología y Antropología Surandinas, n 50, 2015 (pp. 195-213).

43 Quiroz, D., et al., «Campesinos andinos y políticas agrarias durante la Junta de Adelanto de Arica (Azapa, Lluta y la precordillera, 1959-1976)», Idesia, 2011 (pp. 157-168).

${ }^{44}$ Llanque, R. y Villca, E., Qamiris aymaras. Desplazamiento e inclusión de elites andinas en la ciudad de Oruro, PIEB, La Paz, 2011.

45 lbidem, p. 35.

${ }^{46}$ González, S., Sísifo en los Andes la (frustrada) integración física entre Tarapacá y Oruro: las caravanas de la amistad de 1958, RIL, Santiago de Chile, 2012.

47 Llanque, op. cit., p. 55.
} 
citado por Llanque "por ejemplo, yo, con mi poca ganancia que tenía, he llegado a pie a Arica, de alli he regresado a pié también cargando mis cositas, he venido del puerto libre de Arica, traíamos y más barato, después nos traíamos en bicicletas de Arica; además, ese tiempo no habia camiones, no habia caminos, no había nada, todos en bicicleta, en burros, esa ha sido nuestra vida"48.

Entrado los años 70 las tierras de cultivo de los valles de Lluta y Azapa fueron parceladas y vendidas entre chilenos, aymara y no aymara. De ese grupo los aymara bolivianos fueron los que tuvieron menor acceso a la propiedad de la tierra debido a la condición de extranjero y a la imposibilidad jurídica de comprar tierras ${ }^{49}$. Sin embargo, los aymara bolivianos accedieron a la tierra a través de la modalidad de mediería y custodia. La primera modalidad consistía en convenios que implicaban el pago de una renta normalmente en cosechas correspondiente a la mitad de la producción. La segunda corresponde a un acuerdo informal, casi siempre verbal, sobre cuidado y mantención de un predio a cambio de poder explotarlo, esta práctica fue usada especialmente entre parientes ${ }^{50}$. Por ambos sistemas se explica el relevo de aymara chilenos por aymara bolivianos que se produjo en los valles altos, medio y bajo andinos, especialmente notorio en el norte de la región de estudio. En general, este movimiento de población, que se tradujo en cruces frecuentes por la frontera, fue eminentemente rural rural transfronterizo hasta la primera mitad del siglo XX.

\section{Comerciantes y trabajadores bolivianos en Tarapacá de fines del siglo XX}

A fines del siglo XX la movilidad fronteriza boliviana nos remite a la circulación motivada por la búsqueda de trabajo en las ciudades tarapaqueñas y al impulso en el marco de la creación de la Zona Franca de Iquique (ZOFRI) en 1975. La ZOFRI fue la primera zona franca de Chile y fue creada por la promulgación del Decreto Ley 1.055 del 25 de junio de 1975 (ZOFRI, 2007). El objetivo de su creación fue impulsar el desarrollo de la Primera Región a través de la industrialización, instalación de armadurías y la re exportación o re expedición ${ }^{51}$ en un momento en que la región atravesaba por una difícil situación económica. Era la época de las "banderas negras" 52 , crisis arrastrada desde el cierre de las salitreras y luego de fugaz actividad pesquera de los años $60^{53}$. Las primeras operaciones comerciales se "iniciaron en un galpón arrendado en la calle Patricio Lynch, sector de la Puntilla de Iquique, Luego, en el año 1978 se trasladaría al barrio El Colorado, en los suburbios de la ciudad, en un botadero de basuras ubicado sobre extensos arenales donde actualmente opera el Barrio Industrial y las empresas pesqueras elaboradoras de harina de pescado" 54. Los años 90 fueron de desarrollo y expansión de la ZOFRI en Sudamérica y en Chile ${ }^{55}$, actividad que fue estimulada por un mejoramiento de la conectividad entre la región y

\footnotetext{
48 Ibidem, p. 59.

${ }^{49}$ González, Héctor, La posición de aymaras chilenos y bolivianos en la estructura de tenencia de la tierra de los valles de Lluta y Azapa, TEA Taller de Estudios Andinos, Arica, 1998.

${ }^{50}$ Idem.

${ }^{51}$ González, S., «ZOFRI: un enclave comercial», Cuaderno de Investigación Social CIREN, n 14, 1985 (pp. 5-32).

${ }^{52}$ Guerrero, B., op. cit.

${ }^{53}$ Sade, Amin, 31 años de Zofri, publicado el 30 de junio del 2006. Disponible en: http://www.estrellaiquique.cl/prontus4_nots/site/artic/20060630/pags/20060630011348.html

54 ZOFRI, Memoria Anual Zona Franca de Iquique S. A Plataforma de negocios del Cono Sur, ZOFRI S.A, Iquique, 2007, p. 7.

55 En 1991 las utilidades de ZOFRI llegaron a 5 mil 86 millones de pesos que sólo fue superado en 1995 cuando facturó 5 mil 839 millones de pesos. En: La historia de la Zona Franca de Iquique, publicado el 11 de diciembre de
} 
Bolivia ${ }^{56}$, los beneficios tributarios para la salida de mercadería hacia Bolivia y la existencia de un comercio atractivo para los comerciantes bolivianos. En ambos casos encontramos patrones de circulación que se traducen en idas y venidas frecuentes con el objeto de aprovechar la ventaja de los cruces con fines comerciales y también laborales, entre ellas las actividades comerciales, las labores de albañilería, el servicio doméstico y los trabajos agrícolas. Las brechas de desarrollo se traducen en un delta salarial para quienes cruzan la frontera porque según los datos "el año 2011, el PIB per cápita de Bolivia es solo un 30\% del PIB per cápita de Chile"57.

La movilidad desplegada a partir de los años 90 se superpone a las históricas del siglo XX, pero con particularidades distintas que conectan lo regional, con lo nacional y lo global. A la motivación laboral se suma la comercial de los qamiris (ricos aymara) y comerciantes en general que encontraron en el transporte y la compra de productos de la ZOFRI una oportunidad de movilidad social. La mejoría en la conectividad carretera entre Iquique -Oruro, la expansión de empresas de autobuses y camiones que cubren dichos tramos facilitó los traslados y los fletes. Asimismo, la ubicación marginal de Oruro respecto del eje central de Bolivia y su cercanía con la frontera "resultó ser el punto de estratégico importante para los qamiris del Occidente, por su ubicación geográfica y la conexión con la costa del Pacífico, por las carreteras internacionales Oruro Pisiga y también Oruro Patacamaya Tambo Quemado Arica"58.

Al trajín fronterizo se sumó el que viene a la ZOFRI a comprar que se traduce en un movimiento constante de buses y camiones por el paso fronterizo de Colchane. Estos movimientos son semanales, quincenales y mensuales y han dado vida al Barrio Boliviano en Iquique formado por agencias de buses, pensiones de bajo costo, centrales de llamados y negocios de comida boliviana ${ }^{59}$. Lo/as comerciantes bolivianos buscan comprar todo tipo de productos, desde ropa, zapatillas, productos electrónicos hasta vehículos para luego regresar a Bolivia y venderlos en ferias y comercio de Oruro, La Paz, Cochabamba e incluso de ciudades amazónicas como Cobija

\footnotetext{
2011. Disponible en http://www.nortino.com/2011/12/la-historia-de-la-zona-franca-de_11.html

56 Hasta la división de Tarapacá el año 2007 la región compartía casi $600 \mathrm{~km}$ con Bolivia. En la actualidad un elemento central para comprender los movimientos de población fronteriza es la conectividad por tierra existente en la zona a través de tres pasos fronterizos el de Visviri, Chungará-Tambo Quemado y el de ColchanePisiga. Las primeras unen el extremo norte de la región con el sudoeste de Bolivia a una altura de $4096 \mathrm{msnm}$. y $4680 \mathrm{msnm}$. respectivamente. Ambos pasos vinculan a las ciudades de La Paz y Arica con una distancia de 580 y $500 \mathrm{kms}$ en cada caso. La segunda está ubicada a $262 \mathrm{~km}$ de Iquique a una altura $3695 \mathrm{msnm}$ y une a la capital regional de Tarapacá, Iquique con la capital de departamento de Oruro, que lleva el mismo nombre. Si bien estos caminos ya existían antes del periodo de este estudio, su pavimentación y arreglos sólo se concretaron a fines de los años noventa, en el caso de Tambo Quemado, y entrado el siglo XXI para el caso de Colchane. Este último paso y carretera que une Iquique y Oruro es la concreción de una aspiración de casi un siglo de los habitantes de ambas ciudades especialmente porque Oruro ha quedado marginada del eje central formado por La Paz, Cochabamba y Santa Cruz.

57 Juarez, I., El Alto, trampolín de los migrantes rurales de La Paz al exterior, publicado el 6 de marzo de 2018. Disponible en http://www.paginasiete.bo/sociedad/2018/3/6/alto-trampoln-migrantes-rurales-exterior172064.html

${ }^{58}$ Llanque, op. cit., p. 71.

59 Pereira, A. y Uribe, A., Migración comercial boliviana. Acercamiento exploratorio y descriptivo sobre el fenómeno de la migración comercial boliviana y su vinculación con la Zona Franca de Iquique, en la comuna de Iquique. Año 2009, Universidad Arturo Prat, Iquique, 2010; Tapia, M. y Chacón, F., «Vínculos transfronterizos: vida, movilidad y comercio en el Barrio Boliviano de Iquique, Chile», REMHU, Revista Interdisciplinar de la Movilidad Humana, $\mathrm{n}^{\circ} 24$, 2016 (pp. 131-152).
} 
donde también existe una zona franca vinculada comercialmente a la ZOFRI. Este comercio ha incentivado a los comerciantes aymara de Oruro y La Paz para aprovechar las oportunidades del comercio de zona franca y proveer a las principales ciudades bolivianas e incluso incursionar en el comercio internacional con la importación directa desde China, Asia y Estados Unidos. Como señala Llanque "esto generó nuevas actividades en los qamiris de la generación del 90 y surgió una nueva generación, la del 2000; esos importadores eran los hijos de los viejos qamiris del transporte, y del comercio y desarrollaban sus actividades en el sector comercial como una suerte de manejo de pisos -económicos"60.

Respecto al marco jurídico que regula el ingreso y salida el país y la región de Tarapacá la normativa sobre migración y extranjería está dada por la Ley de Inmigración del año 1975 por el que las autoridades regionales gestionan los flujos migratorios, especialmente de origen sudamericano. A partir de la firma del Tratado de Paz, Amistad y Comercio de 1904 con Bolivia se establecieron las relaciones de vecindad marcadas por el libre derecho de tránsito comercial por los territorios de las regiones nortinas y los puertos del Pacífico, entre ellos Arica y Antofagasta y recientemente Iquique. En general a partir del Tratado y las normativas sucesivas han privilegiado las relaciones comerciales y el libre tránsito a los bienes y mercancías y ha quedado en un segundo lugar el tránsito de personas. A pesar de la existencia de "la Convención sobre Tránsito de 1937 que reconoció el derecho de libre tránsito en todo tiempo, sin excepción alguna, e incluyó a las personas", en general la población Bolivia se acoge a la Ley de Extranjería de 1975. Sin embargo, en el último tiempo por el Acuerdo de Residencia de los Nacionales de los Estados Partes del MERCOSUR Bolivia y Chile de 2009 los nacionales de Argentina, Bolivia, Brasil, Paraguay y Uruguay se les podrán otorgar visaciones de residencia temporaria por un año prorrogable por igual periodo (Oficio Circular $\mathrm{N}^{\circ}$ 26465, de Subsecretario del Interior, de 04.12.09)

\section{Motivos, expectativas y estrategias de cruce fronterizos a fines de los años 90 y principios del siglo XXI}

En el marco de las migraciones recientes hacia Chile nos interesa revisar en este apartado los motivos, expectativas y estrategias de cruce fronterizos levantados en la información de prensa. Como señalamos más arriba la contigüidad de Tarapacá y Bolivia, el mejoramiento de las carreteras, las diferencias de desarrollo y la atracción que provoca la actividad económica explican la llegada y circulación de trabajadores y comerciantes bolivianos. En el contexto de las recientes migraciones, Tarapacá ha sido una de las regiones que mayor crecimiento económico ha experimentado en las últimas décadas y por tanto un lugar atractor de migración. Entre 1990 y 2004 Chile creció a una tasa del 5\% y la región en estudio lo hizo en un $6 \%$, un punto más que el nacional ${ }^{61}$. Por tanto, Tarapacá, junto a Antofagasta y Atacama, presentan un gran dinamismo económico gracias a la minería lo que se traduce en una mayor demanda de servicios. La actividad minera sumada a la actividad portuaria y comercial ha actuado como atractor de población interna y fronteriza convirtiendo

\footnotetext{
${ }^{60}$ Durán, C., et al., Migrantes, paisanos y comerciantes. Prácticas sociales y económicas en la Zona Franca de Cobija (1998-2011), Programa de Investigación Estratégica en Bolivia PIEB, La Paz, 2013.

${ }^{61}$ Bellolio, Á. y Errázuriz, H., Migraciones en Chile. Oportunidad ignorada, Ediciones LYD, Santiago de Chile, 2014.
} 
a la región de Tarapacá en una de las de mayor crecimiento demográfico en las últimas décadas ${ }^{62}$.

Estos factores permiten pensar en desplazamientos de corto plazo o acotadas a objetivos específicos, trabajo y comercio, cuya movilidad no implica el establecimiento de largo alcance. Por tanto, se tipifica una circulación de personas de duración variable que da lugar en algunos casos la instalación en la región sin necesariamente dejar de viajar o circular. En este sentido las estrategias de cruce como turistas se entienden en tanto se evita el trámite y los gastos de obtención de pasaporte. El incentivo para este tipo de modalidad son las expectativas económicas y las noticias de que en Chile hay trabajo y que los salarios son mayores que en Bolivia. Al respecto una nota del diario La Estrella de Iquique sobre tramitación de visas en la Gobernación de Iquique señala que:

"Las personas que hablaron dijeron que Iquique cumplia con las expectativas que ellos se habian hecho con respecto a la situación laboral y económica. Así lo dijo Celsio Flores quien es boliviano y tiene residencia en Pica. 'Ahora estoy haciendo algunos trámites, pero estoy muy bien cuanto a lo económico y no he tenido problemas con la gente'

"Zara Maison, asesora del hogar, de nacionalidad boliviana, tramitaba su residencia sujeta a contrato y dice que en Chile se paga mucho más que en mi país, por lo tanto, las expectativas se me han cumplido"

"Carlos Miranda, proveniente de un pueblo fronterizo de Bolivia, dijo q: 'vine de visita a Iquique y me gustó, por lo que me quiero quedar para trabajar, Ahora quiero comenzar a hacer los trámites de autorización"

"Casi todos dijeron que el Gobierno chileno da muchas facilidades para hacer los trámites, sólo que estos demoran mucho, obligando a algunos a salir del país" (La Estrella de Iquique 12 de febrero 1998).

La circulación de extranjeros fronterizos en Tarapacá ha dado lugar, en algunos casos, al establecimiento en la región por razones laborales, lo que no implica el fin del vínculo con las zonas de origen, como vimos para el caso de Arica, sino al surgimiento de modos de vida binacional. En general se aprecian prácticas de idas y vueltas por tiempos variables y en los que la regularidad e la irregularidad jurídica ayuda a comprender en muchos casos la precariedad laboral y la vulneración de los derechos de los extranjeros ${ }^{63}$. La labor de algunos dispositivos de acogida, como es el caso del Servicio Jesuita Migrante de Tacna y de Arica y la Pastoral Migratoria de Iquique, permiten paliar de alguna forma las dificultades a las que se ven enfrentados los extranjeros que

\footnotetext{
${ }^{62}$ La región de Tarapacá ocupa el primer lugar en crecimiento intercensal (2002-2017) a nivel regional con una estimación de un 30\% aproximado según datos del censo 2017 (www.censo2017.cl).

63 Tapia, Marcela, «Extranjeros fronterizos en las regiones extremas de Chile: entre migración y circulación. 19902014", en Rojas, N. y Vicuña, J.T. (eds.), Migración y Trabajo. Estudio y propuestas para la inclusión sociolaboral de migrantes en Arica. Ciudadano Global /OIM, Santiago, 2014 (pp. 31-55).
} 
llegan a la región y contener de alguna forma a quienes apuestan por el cruce de las fronteras ${ }^{64}$. Respecto de estas dinámicas de movilidad la prensa consigna lo siguiente en 1998:

\begin{abstract}
Más de 25 ilegales en la región. La Estrella de Iquique, 3 marzo de 1998 "Un incremento en la presencia de bolivianos y peruanos en la Primera región, principalmente en Iquique y Arica, está ocasionando serios problemas sociales y laborales en la zona.

Ello pese a que muchos extranjeros de estas nacionalidades son hombres $y$ mujeres que viven honradamente y en forma legal.

Un grupo considerable trabaja como asesoras del hogar y en la construcción.

Pero un grupo importante se involucra en problemas con la justicia y son numerosos los que no tienen documentos legales para permanecer en el país.

Este último grupo lo integran quienes pasan a Chile a través de una zona limitrofe que no es respetada. Llegan a Iquique en camiones y otros tipos de vehículos. Para no despertar sospechas trabajan un tiempo en algún pueblo rural o altiplánico. Y cuando adquieren confianza a las ciudades capitales de las provincias de Iquique y Arica" (La Estrella de Iquique, 3 de marzo de 1998).
\end{abstract}

En general, estos movimientos de población han sido menos notorios y de tipo preferentemente rural rural transfronterizo, y por lo mismo silenciosos, sin embargo su importancia se refleja en los datos debido a la progresiva pérdida de población de los poblados del interior de la región. Según cifras recientes del INE aparecidos en la prensa señalan que la proporción de bolivianos respecto del total de extranjeros en la Provincia del Tamarugal es de un $80 \%$, la mayoría en situación de irregularidad ${ }^{65}$. Esta migración tiene mayor asentamiento, pero mantiene prácticas de movilidad binacional, es decir, en frecuente movimiento entre Chile y Bolivia.

La prensa informa diversas prácticas de ingreso de bolivianos a Tarapacá en las que predomina el cruce por pasos no habilitados que, para la época de estudio, fueron tipificados como ilegales. La motivación de estos cruces es fundamentalmente la búsqueda de trabajo en especial para incorporarse en las actividades agrícolas de Azapa y Lluta al norte de la región, a los distintos valles alto andinos, el oasis de Pica y a las ciudades costeras de Arica e Iquique. El año $1990 \mathrm{La}$ Estrella de Arica informa que 23 ciudadanos bolivianos fueron sorprendidos trabajando en la región "en diversas parcelas del valle de Azapa y Lluta y su ingreso al territorio nacional fue por

\footnotetext{
64 Tapia, M. y Ramos, R., «Mujeres migrantes fronterizas en Tarapacá a principios del siglo XXI. El cruce de las fronteras y las redes de apoyo», Polis. Revista Latinoamericana, $n^{\circ} 12$ (35), 2013 (pp. 229-257).

65 Davied, Jaime, Familias bolivianas se instalan en Tarapacá, arriendan tierras y reactivan el agro altiplánico, publicado en Emol el 10 de agosto de 2014.

Disponible en http://impresa.elmercurio.com/Pages/NewsDetail.aspx?dt=2014-08-10\&dtB=09-092014\%200:00:00\&Paginald $=12 \&$ bodyid $=3$
} 
pasos no habilitados y por las noches, según manifestaron al ser interrogados sobre su ingreso clandestino al país... Los bolivianos llegaron a esta zona a trabajar en labores agrícolas cambiándose cada cierto tiempo de lugar a fin de evitar ser sorprendidos por su ingreso ilegal" (La Estrella de Iquique, 21 de abril de 1990).

El cruce por pasos no habilitados es recurrente, especialmente para los aymara bolivianos que habitan comunidades cercanas a la frontera y que buscan instalarse en localidades fronterizas como Visviri o Chungará, entre otras. Las entradas y permanencias temporales o definitivas pueden durar años hasta que son sorprendidos en controles de identificación a buses o transeúntes que circulan desde o hacia dichas localidades. Para evadir el control policial la prensa informa de algunas estrategias, como la de moverse de una localidad a otra o ingresar con ropa de "infantes" tal como lo señala La Estrella de Iquique en una diligencia hecha por carabineros en junio de 1992 en la que fueron aprendidos dos agricultores bolivianos. "Ambos caminaban de infantes A 123 cuando fueron sorprendidos y detenidos por personal de Carabineros de Retén de Caquena dependiente de la Tenencia Chungará, ya que al solicitarles su identificación respondieron que no contaban con documentos personales ni autorización para permanecer en territorio chileno, reconociendo que habian ingresado en forma ilegal a nuestro país" (La Estrella de Arica, 9 de junio de 1992).

Quienes logran evadir el control policial y permanecen en la región sin documentos corren el riesgo de ser detenido en las ciudades costeras de Arica e Iquique a las que acceden luego de varias idas y venidas alentados por mayores ofertas de trabajo. En un caso que consigna La Estrella de Arica de 1993 una mujer boliviana compró una cédula de identidad a un chofer de bus del recorrido Arica La Paz

"En 1990 la mujer ingresó en forma ilegal a Chile a través de un paso no habilitado cercano a Visviri, con el fin de buscar mejores perspectivas económicas, dada la pobreza en que vivía su familia.

En Arica trabajó en distintas casas particulares sin permanecer mucho tiempo en un lugar, por temor a que descubrieran su carácter indocumentado y pudieran denunciarla a la policía.

Cansada de sufrir esta situación inestable, recientemente decidió volver al país vecino. Sin embargo, por su ingreso irregular tampoco podía asegurarse un retorno sin dificultades... Entonces se contactó con Elfren Friedler, chofer de una máquina del recorrido Arica La Paz a quien le adquirió la cédula de identidad chilena" pero fue detenida en Poconchile" (La Estrella de Arica, 21 de enero de 1993).

Otra práctica de cruce consignada en la prensa es el paso regular por controles fronterizos en calidad de turista. Como señala la legislación chilena los turistas no están habilitados para realizar actividades remuneradas, sin embargo, es un recurso utilizado frecuentemente para llegar a los centros urbanos de la región e incorporarse en el mercado de trabajo informal. En otro estudio encontramos que es frecuente, entre mujeres bolivianas, ingresar a la región como turistas, emplearse como asesora del hogar puertas adentro y una vez vencido el tiempo de permanencia como turista (90 días) "hacen frontera", es decir, viajan hasta Colchane cruzan a Pisiga ahí estan

INTUS-LEGERE HISTORIA/ ISSN 0718-5456| EISSN 0719-8949/Año 2018, Vol. 12, № 1, pp. 66-86. 
allí un día o viajar a su localidad de origen y luego vuelven a entrar al país. Con ello se activan nuevamente otros 90 días y no pierden el vínculo laboral en la región.

Estas prácticas de cruces por pasos no habilitados o de entrada como turistas para trabajar despertaron la preocupación de las autoridades regionales y de los gremios de trabajadores. Esta inquietud se expresó en la prensa local a través de notas editoriales y cartas al editor como la siguiente:

"El ingreso y egreso de personas a Chile está regulado por un reglamento de extranjería. A su vez, en las cercanías de los límites fronterizos existen recintos habilitados que controlan la salida y entrada de personas del territorio nacional. Sin embargo, para nadie es un misterio que la zona norte posee extensas fronteras que son muy fáciles de vulnerar. Es así como llegan al país una serie de personas sin documentación en busca de trabajo y mejores expectativas. Este flujo afecta la mano de obra nacional y no es fácil de cuantificar.

El presidente provincial de la Central de Trabajadores, Néstor Jorquera, indicó que la cantidad de extranjeros indocumentados presentes en las faenas productivas es indeterminada, pero en el último tiempo se ha incrementado. Opinión similar tienen en la Gobernación y en la Policía de Investigaciones.

A los indocumentados hay que agregar las personas que ingresan con visa de turista y trabajan. Los extranjeros que se desempeñan en faenas de la construcción, agrícolas o de Zona Franca, a las cuales se suman labores de asesoras del hogar. Obviamente por encontrarse en una situación irregular ofrecen su trabajo e un menor costo" (La Estrella de Iquique 1 de noviembre de 1995).

Según datos del censo 2017 recientes las actuales regiones de Arica y Parinacota y de Tarapacá tienen un $8 \%$ y un $14 \%$ de población extranjera respecto al total de su población. En el primer caso la población boliviana corresponde al $43,2 \%$ del total de los extranjeros y en el Tarapacá es un $44,4 \%$ del total de los extranjeros seguido por los peruanos ${ }^{66}$. Por tanto ambas regiones poseen la mayor proporción de extranjeros de origen boliviano, lo que da cuenta de la profundidad histórica que tiene la presencia de bolivianos en el extremo norte del país. Esta situación es el resultado de las dinámicas antes descritas y todo indica que seguirá en aumento.

\section{Conclusiones}

La presencia de bolivianos en la región de Tarapacá y los patrones de movilidad que han descrito en la historia del siglo XX y entrado XXI muestran que el norte de Chile es y ha sido parte del campo migratorio de ese país, el que ha pasado de ser marginal pero constante en el siglo XX , a una tendencia de seguir creciendo en la actual centuria. Los aportes de la geografía social francesa y del paradigma de la movilidad son centrales para comprender este campo migratorio en tanto permite mirar no sólo los migrantes en sentido estricto, es decir, aquellos que se establecen

\footnotetext{
${ }^{66}$ www.censo2017.cl
}

INTUS-LEGERE HISTORIA/ ISSN 0718-5456| EISSN 0719-8949/Año 2018, Vol. 12, № 1, pp. 66-86. 
y se quedan, sino detenernos en aquellos que se mueven a través de la frontera con fines laborales y comerciales. Se trata de personas, trabajadores temporales, jornaleros agrícolas o comerciantes que buscan traspasar la frontera para ampliar las fuentes de recursos o para cumplir con un proyecto aprovechando las ventajas del cruce y los ciclos económicos. Por otro lado, la mirada diacrónica de la movilidad en la región fronteriza permite superar el presentismo que a veces padecen los estudios migratorios y el carácter de novedad que le dan a la nueva migración como si se tratase de un fenómeno totalmente nuevo. Así es posible ver cómo las prácticas sociales fronterizas son históricas y realizadas por la población boliviana y aymara a las que se superponen otras más recientes constituyéndose en un acervo o un saber circular que se actualiza en el presente.

De los años 90 a la fecha, los movimientos de población se enmarcan en el contexto del aumento de la migración sudamericana y caribeña a Chile y se conecta con experiencias migratorias previas a otros destinos de la migración boliviana. Éstas últimas se han desprendido del carácter regional de los movimientos que tenían por escenario las zonas más cercanas a la frontera y eventualmente las ciudades costeras durante el siglo XX para extender su área de influencia gracias al auge de la economía regional y las posibilidades que da la ZOFRI en un contexto de inserción internacional regional y porque no decir de la globalización. La crisis en el sur de Europa y en Estados Unidos, los traspiés de las economías regionales, las bajas expectativas salariales en el país de origen y la insustentabilidad de la actividad agrícola y ganadera alto andina ha motivado el aumento de la movilidad boliviana con fines laborales hacia Tarapacá. Ello sumado a la necesidad de mano de obra en nichos específicos de trabajo de bajo estatus y escasa aceptabilidad para los nacionales han hecho de la región una opción para la búsqueda de nuevas posibilidades laborales y económicas.

La inclusión de la noción de región fronteriza favorece la observación de los fenómenos y prácticas sociales de las personas que pasan las fronteras porque ocurren en lugares concretos y unen con sus trayectorias dos o más estados. Por tanto no hay que olvidar que la migración $\sim$ y movilidad en general existen en la medida que hay lugar o lugares a los que se pertenece y de los que se ha partido, pero que no necesariamente dan lugar a de procesos de integración, establecimiento o búsqueda del estatus de ciudadanos como fin último. Así es posible captar movimientos de población fronteriza de temporalidad variable más allá de la migración laboral con asentamiento definitivo que ha predominado en los estudios migratorios. Junto a la noción de región fronteriza es posible comprender cómo la frontera se convierte en un recurso incluso en contextos diplomáticos complejos como ocurre en el caso estudiado.

La inclusión de la larga duración en el análisis permite develar los movimientos silenciosos de aymaras bolivianos que han quedado apartados de los estudios migratorios, pero que han tenido su contraparte en los estudios antropológicos. La puesta en contexto en clave de movilidad permite ampliar la comprensión de estos movimientos y comprenderlos como un saber circular histórico que se traduce en prácticas sociales fronterizas que unen regiones colindates y producen transfrontericidad, es decir, interacción humana por movilidad y migración donde la frontera es la clave explicativa.

INTUS-LEGERE HISTORIA/ ISSN 0718-5456| EISSN 0719-8949/Año 2018, Vol. 12, №1, pp. 66-86. 


\section{Bibliografía:}

- Ariostegui, J., (1998) "Historia y Tiempo Presente. Un nuevo horizonte de la historiografía contemporaneista" en Cuadernos de Historia Contemporánea, 20, 15 18.

- BÉDARIDA, F., (1998) "Definción, método y práctica de la Historia del Tiempo Presente" en Cuadernos de Historia Contemporánea 20, 19 27.

- Benedetti, A. y SAlizzi, E., (2011) "Llegar, pasar, regresar a la frontera. Aproximación al sistema de movilidad argentino boliviano" en Transporte y territorio, 4, 148 179.

- Bertaux, D., (1993),Desde la perspectiva de la historia de vida a la transformación de la práctica sociológica, En Marinas, J. M. y. C. S. (Ed.), La Historia oral: métodos y experiencias. Madrid: Debate, 1993, pp. 19 34

- Burke, P., (1987) Sociología e Historia, Madrid: Alianza Editorial, 1987

- _. (2009) Formas de hacer historia, Madrid: Alianza Editorial, 2009

- Cortés, G., (2009) "Migraciones, construcciones transnacionales y prácticas de circulación. Un enfoque desde el territorio" en Párrafos geográficos, 8 (1), 35 53.

- Davied, J. (10 de agosto de 2014). Familias bolivianas se instalan en Tarapacá, arriendan tierras y reactivan el agro altiplánico. Emol.

- Domenach, H., et al., (2007) Movilidad y procesos migratorios en el espacio fronterizo Argentino Bolviana, Córdoba: Editorial Copiar, 2007

- González, H., (1998) La posición de aymaras chilenos y bolivianos en la estructura de tenencia de la tierra de los valles de Lluta y Azapa, Arica: TEA Taller de Estudios Andinos, 1998

- Jessop, B., (2004) "La economía política de la escala y la construcción de las regiones fronterizas" en EURE, 30 (89), 25 41.

- Johnson, C., et al., (2011) "Interventions on rethinking 'the border' in border studies" en Political Geography, 30, (2), 61 69.

- Mallimaci, A. I., (2012) "Movilidades y permanencias. Repensando la figura del movimiento en las migraciones" en Revista de Temas de Antropología y Migración, 77 92.

- Nweined, K., (1992) Frontera y límite en su marco mundial: una aproximación a la "fronterología", Caracas: Ediciones de la Universidad Simón Bolívar 1992

- PAASI, A., (2005) "Generations and the 'Development' of Border Studies" en Geopolitics, $10,(4), 663 \sim 671$.

- Rioux, J. P., (1998) "Historia del Tiempo Presente y demanda social" en Cuadernos de Historia Contemporánea, 20, 71 80.

- Sassen, S., (2010) Territorio, autoridad y derechos. De los ensamblajes medievales a los ensamblajes globales, Madrid: Katz Editores, 2010

- Sauvage, P., (1998) "Una historia del tiempo presente " en Historia Crítica, 17, 59 70.

- TAPIA, M., (2013),Migración y movilidad de los trabajadores fronterizos en Tarapacá durante el ciclo del nitrato. 1880 1930, En González, S. (Ed.), La sociedad del salitre: 
protagonistas, migraciones, cultura urbana y espacios públicos, 1870 1940. Santiago de Chile: RIL, 2013, pp. 163 194

- Tapia, M. y GonzÁlez, A., (2014),Presentación. Fronteras, regiones fronterizas y migraciones. Entre apertura, integración y cierre, En Tapia, M. y A. González (Eds.), Regiones fronterizas, migración y los desafios para los Estados nacionales latinoamericanos. Santiago de Chile: RIL Editores, 2014, pp. 15 38

- TARrius, A., (2000) "Leer, describir, interpretar las circulaciones migratorias: conveniencia de la noción de 'territorio circulatorio'. Los nuevos hábitos de la identidad" en Relaciones, 21 (83), 39 66.

- _. _. (2001) "Au delà des États nations : des sociétés de migrants " en Revue européenne de migrations internationales, 17 (2), 37 61.

- _. (2010) "Pobres en migración, globalización de las economías y debilitamiento de los modelos integradores: el transnacionalismo migratorio en Europa meridional" en Empiria. Revista de metodología de Ciencias Sociales, 19, 133 156.

- Texidó, E. y Gurrieri, J., (2012) Panorama migratorio de América del Sur 2012, Buenos Aires: Organización Internacional para las migraciones OIM, 2012

- Zapata Barrero, R. y Ferrer Gallardo, X., (2012), Las fronteras en la época de la movilidad, En Zapata Barrero y X. Ferrer Gallardo (Eds.), Fronteras en movimiento. Migraciones hacia la Unión Europea en el contexto Mediterráneo. Barcelona: Edicions Bellaterra, 2012, pp. 11 56 